\title{
Precocidade sexual de novilhas Nelore em rebanho sob seleção no Estado do Acre
}

\author{
Sexual precocity of Nellore heifers in a herd under selection in the State of Acre, Brazil \\ Precocidad sexual de novillas Nellore em um rebaño selección em el Estado de Acre
}

Recebido: 16/03/2021 | Revisado: 26/03/2021 | Aceito: 26/03/2021 | Publicado: 04/04/2021

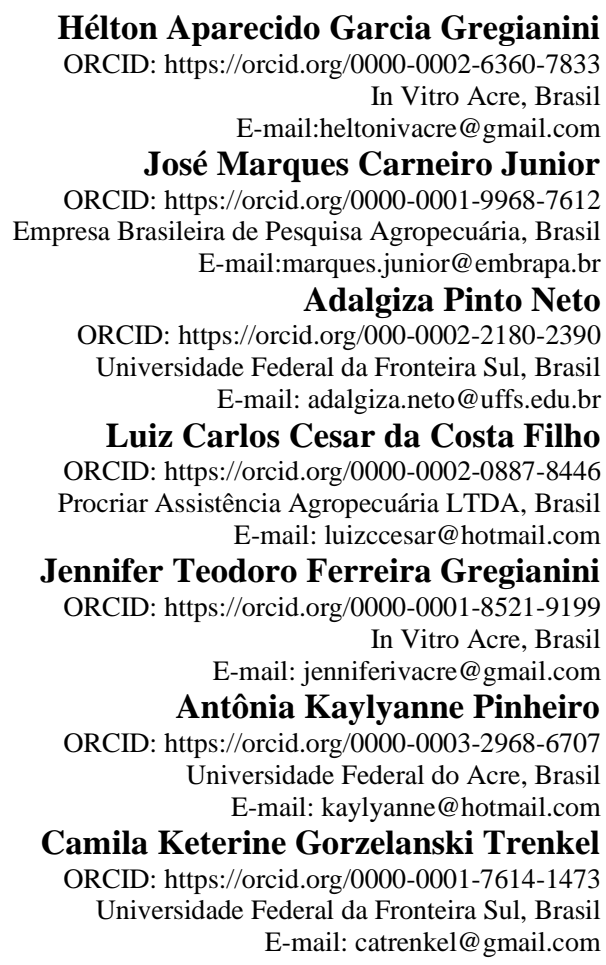

\section{Resumo}

Avaliou-se a precocidade sexual de novilhas em programa de melhoramento genético no Acre, entre janeiro/2018 a fevereiro/2019, onde 184 novilhas Nelore com idade de 11-14 meses, peso médio ajustado no momento da indução de ciclicidade (IC) de $280,17 \mathrm{Kg}$ e ganho de peso médio de $0,6 \mathrm{Kg} /$ dia, foram pesadas, submetidas a protocolo de IC e classificadas em Categoria A (tônus uterino e ciclicidade ovariana), Categoria B (tônus uterino ou ciclicidade) e Categoria C (acíclicas e sem tônus uterino), e submetidas a sincronização do estro visando IATF, com diagnóstico de gestação realizado após 30 dias. Foram ainda submetidas à ultrassonografia de carcaça para obtenção da área de olho de lombo (AOL) e acabamento de carcaça (ACAB). Analisou-se: AOL, ACAB, peso, índice de seleção, idade, taxa de gestação e correlação de Pearson entre as variáveis peso, AOL, ACAB, índice de seleção e idade. A taxa de gestação foi de $42 \%$, sendo semelhante entre as categorias ( $>>0,05)$. A AOL e ACAB não apresentou diferença entre novilhas gestantes e não gestantes $(\mathrm{p}>0,05)$. Diferenças foram observadas entre as categorias para as variáveis idade e peso ajustado ao dia da IC ( $p<0,05)$, não sendo observado diferenças para $\mathrm{AOL}$ e $\mathrm{ACAB}(\mathrm{p}>0,05)$. Não foram observadas diferenças entre o potencial genético dos touros utilizados. Conclui-se que o rebanho estudado possui elevada precocidade sexual para as novilhas Nelore, super-precoces e precoces respectivamente, e aquelas que apresentaram maior peso tenderam a ser mais precoces, indicando a importância desta característica em sistemas de produção.

Palavras-chave: Carcaça; Indução sexual; Peso à puberdade; Reprodução; Super-precoce.

\begin{abstract}
The sexual precocity of heifers of a breeding program in the state of Acre, Brazil, was evaluated from January 2018 to February 2019. The herd included 184 Nellore heifers of 11 to 14 months of age, with mean body weight of $280.17 \mathrm{~kg}$ at the time of cyclicity induction (CI) and mean body weight gain of $0.6 \mathrm{~kg}$ day- 1 . The heifers were weighed, subjected to the CI protocol, and classified into the Categories A (uterine tone and ovarian cyclicity), B (uterine tone or ovarian cyclicity), and $\mathrm{C}$ (ovarian acyclicity and no uterine tone); then, they were subjected to estrus synchronization focused on fixed-time artificial insemination, with pregnancy diagnosis performed after 30 days. They were also subjected to carcass ultrasound to assess the ribeye area (REA) and carcass finishing level (CFL). The variables evaluated were: REA, CFL, body weight, selection index, age, and pregnancy rate. Pearson's correlation was
\end{abstract}


used for the variables body weight, REA, CFL, selection index, and age. The pregnancy rate found was $42 \%$, which was similar $(p>0.05)$ in all categories. Pregnant and non-pregnant heifers presented similar $(p>0.05)$ REA and CFL. The categories presented differences $(p<0.05)$ for age and body weight at CI, and no differences $(p>0.05)$ in REA and CFL. The bulls used presented no difference in genetic potential. The Nellore heifers from the study herd have high sexual precocity, classified as super-precocious and precocious. Heifers that present higher body weight tended to be more precocious, denoting the importance of this characteristic in production systems.

Keywords: Carcass; Sexual induction; Weight at puberty; Reproduction; Super-precocious.

\section{Resumen}

La precocidad sexual de novillas se evaluó en un programa de mejoramiento genético en Acre, Brasil, entre enero de 2018 y febrero de 2019, donde 184 novillas Nellore (Bos indicus) con edades de 11 a 14 meses, con peso promedio ajustado al momento de la Inducción de Ciclicidad (IC) de 280,17 kg y aumento promedio de peso de 0,6 kg por día fueron pesadas, sometidas al protocolo de Inducción de Ciclicidad y clasificadas en Categoría A (tono uterino y ciclicidad ovárica), Categoría B (tono uterino o ciclicidad) y Categoría C (sin ciclicidad y sin tono uterino), y sometidas a la sincronización del estro con el objetivo de realizar una Inseminación Artificial de Tiempo Fijo (IATF), con diagnóstico de embarazo realizado a los 30 días. También se sometieron a examen de ultrasonido de las costillas para obtener el área del ojo de costilla (AOL) y la grasa subcutánea que cubre las costillas (ACAB). Se analizó: área del ojo de costilla, grasa subcutánea, peso, índice de selección, edad, tasa de embarazo y correlación de Pearson entre las variables peso, área del ojo de costilla, grasa subcutánea, índice de selección y edad. La tasa de embarazo fue del $42 \%$, siendo similar entre categorías $(\mathrm{p}>0,05)$. El área del ojo de costilla y la grasa subcutánea no mostraron diferencias entre las novillas embarazadas y las no embarazadas ( $p>0,05)$. Se observaron diferencias entre las categorías para las variables edad y peso, ajustadas al día de la Inducción de Ciclicidad ( $<<0,05)$, no observándose diferencias para el área del ojo de costilla y para la grasa subcutánea $(p>0,05)$. No se observaron diferencias entre el potencial genético de los toros utilizados. Se concluye que el rebaño estudiado tiene alta precocidad sexual para novillas Nellore, superprecoces y precoces, respectivamente, y las que presentaron mayor peso tendieron a ser más precoces, lo que indica la importancia de esta característica en los sistemas de producción.

Palabras clave: Costillas; Inducción sexual; Peso en la pubertad; Reproducción; Superprecocidad sexual.

\section{Introdução}

A pecuária brasileira possui o maior rebanho comercial do mundo, com aproximadamente 221 milhões de cabeças (Abiec, 2018), criados exclusivamente a pasto, possibilitando obter carne de boa qualidade e de alta competitividade no mercado internacional (Abud, 2011).

No Estado do Acre, a pecuária possui grande importância econômica, representado $40 \%$ do valor bruto da produção do setor agropecuário (Sá et al., 2010). Atualmente o Estado possui 3,1 milhões de cabeças de bovinos (Brasil, 2018) criados em uma área de aproximadamente 1,56 milhões de hectares de pastagens (Barbosa et al., 2015). A produtividade na pecuária de corte tem aumentado significativamente nos últimos anos, verificando crescente inserção de estratégias que proporcionem melhores resultados de eficiência produtiva e qualidade dos produtos (Oaigen et al., 2013; Carvalho \& Zen, 2017). Neste sentido a utilização de tecnologias básicas como manejo, sanidade, nutrição e genética tem sido fortemente incrementado nos sistemas de produção (Artmann et al., 2014).

A reprodução também tem sido de fundamental importância econômica, devido ao impacto que a precocidade sexual representa no desempenho lucrativo da bovinocultura de corte (Siqueira et al., 2003). A precocidade sexual da fêmea bovina pode ser mensurada por meio da idade à puberdade ou idade ao primeiro parto, que pode ser definida como a rapidez de desenvolvimento dos tecidos ou taxa de crescimento, que permite atingir determinada proporção do peso adulto e a expressão das características relacionadas com a puberdade (Cartwright, 1970; Lanna \& Packer, 1997).

As características reprodutivas são de grande importância nos sistemas produtivos de gado de corte, pois o desempenho reprodutivo dos animais é um dos fatores determinantes da eficiência total de produção na bovinocultura de corte. Entretanto, estas características são governadas por vários pares de genes, além de apresentarem grande influência das condições de manejo, nutrição e sanidade, o que resulta em baixos valores de herdabilidade (Alencar, 2002).

Estima-se que 87,7\% do rebanho de corte brasileiro seja de origem zebuína, dentre os zebuínos, 78\% (121,6 milhões) têm a raça Nelore em sua composição genética predominante (Rosa et al., 2016). Dentre os animais zebuínos inseridos em 
programas de melhoramento com seleção para precocidade sexual, as fêmeas jovens podem atingir a puberdade entre 13 e 15 meses de idade. Já em fêmeas zebuínas criadas extensivamente, sem programa de melhoramento, a puberdade varia entre 22 e 36 meses de idade, cuja idade ao primeiro parto irá ocorrer em média aos quatro anos (Souza et al., 1995; Silva et al., 2018).

A tendência atual de mercado está voltada para a utilização de animais precoces, resultando em maior viabilidade econômica, por permanecerem menos tempo em recria, encurtando o ciclo de produção. Isso permite maior velocidade de reposição devido à entrada de animais jovens e de maior potencial genético ao rebanho, proporcionando maior pressão de seleção no rebanho, tornando o sistema cada vez mais produtivo e rentável (Jorge Junior et al., 2001; Boligon \& Albuquerque, 2010).

Diante do exposto, objetivou-se com esse estudo avaliar a precocidade sexual de novilhas Nelore, inseridas em programa de melhoramento genético, no Estado do Acre.

\section{Metodologia}

Este estudo foi realizado na Fazenda Itaituba, inserida em programa de melhoramento genético, localizada no Município de Bujari, Estado do Acre, em parceria com a In Vitro Acre, localizada em Rio Branco, Acre, mediante pesquisa de campo quantitativa (Pereira et al., 2018). Para tanto, 184 novilhas da raça Nelore, de mesmo grupo de manejo, com idade entre 11 e 14 meses, peso médio de $262 \mathrm{Kg}$ e média ponderal de ganho peso de 0,6 Kg/dia, foram inseridas nesse estudo, que ocorreu durante o período de janeiro de 2018 a fevereiro de 2019. Após desmama, todas as novilhas foram mantidas a pasto, recebendo sal proteinado de baixo consumo, avaliadas e dividas em três categorias: categoria A (novilhas aptas a reprodução, com tônus uterino e ciclicidade ovariana), categoria B (novilhas com tônus uterino ou ciclicidade ovariana) e categoria C (novilhas acíclicas e sem tônus uterino).

Todas as novilhas foram submetidas a um protocolo hormonal visando indução da ciclicidade (IC), com progesterona aplicada no dia zero (150mg de progesterona injetável, Sincrogest ${ }^{\circledR}$, Ouro Fino, Brasil) e estradiol, no dia $10(0,5 \mathrm{mg}$ de Cipionato de Estradiol, SincroCP®, Ouro Fino, Brasil). No início desse protocolo administrou-se suplementação mineral injetável (5mL, Biogêneses Bagóß), vacinas contra IBR, BVD e Leptospirose (Bioabortogen e Bioleptogen Biogêneses Bagóß), e realizou-se a pesagem.

Aproximadamente um mês após o protocolo de IC (meados agosto 2018), iniciou-se um protocolo de sincronização de estro e ovulação, visando IATF em todos os animais experimentais (Figura 1), e após 30 dias da inseminação, realizou-se o diagnóstico de gestação, através da ultrassonagrafia transretal (Mindray DP10).

Figura 1. Protocolo hormonal utilizado para sincronização de estro e ovulação visando IATF nas novilhas experimentais.

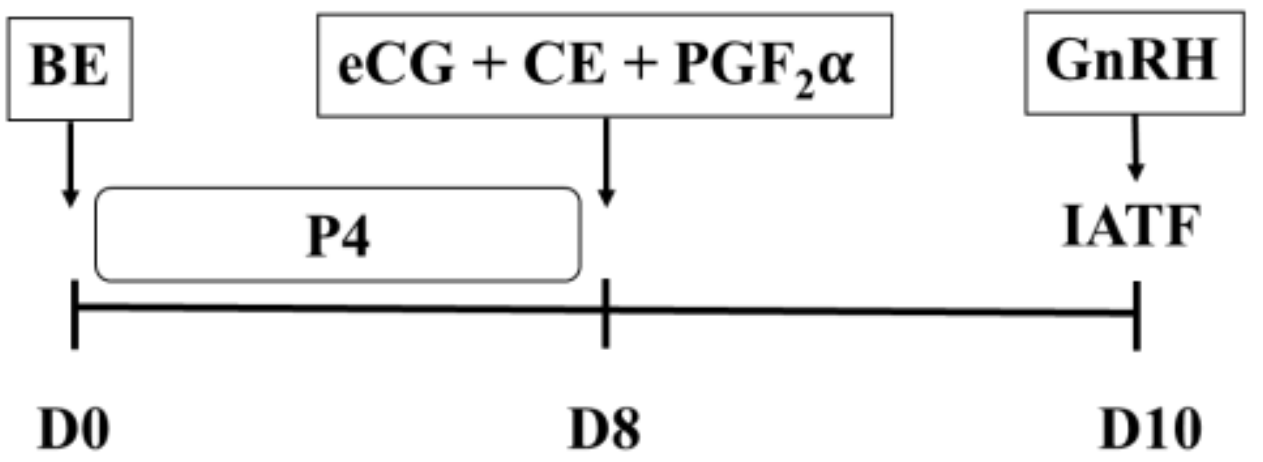

Legenda: D0: Dia zero; D8: dia oito; D10: Dia dez; BE: Benzoato de estradiol (1mL. Bioestrogen $®)$; P4: implante monodose de 1mg progesterona intravaginal (1mg. Cronipress Monodose BiogenesesBagóß); eCG: Gonadotrofina Coriônica Equina (150UI. ECGON®, BiogénisisBagó®); CE: Cipionato de Estradiol (1mL. CRONI-CIP®, BiogénisisBagó ${ }$, Brasil); PGF2 $\alpha$ : Prostaglandina (CRONIBEN®, Biogénisis, Brasil); GnRH: Hormônio Liberador de Gonadotrofina (GONAXAL®, BiogénesisBagó®, Brasil) e IATF: Inseminação Artificial em Tempo Fixo. Fonte: Fernades et al. (2016). 
No final do período experimental (fevereiro 2019) as novilhas foram submetidas à avaliação ultrassonográfica para avaliação de carcaça, onde se mensurou área de olho de lombo (AOL), acabamento de carcaça (ACAB), peso e idade. As novilhas agrupadas nas categorias A, B e C foram comparadas em relação ao diagnóstico de gestação, idade, peso ajustado em relação ao início da IC, AOL e ACAB e índice de seleção (composto por 50\% para peso, 25\% para área de olho de lombo e $25 \%$ para acabamento). Foi realizada Correlação de Pearson destas variáveis com as informações obtidas na ultrassonografia de carcaça.

As novilhas gestantes foram classificadas como super-precoces ou precoces de acordo com a idade a primeira concepção. Assim, novilhas que ficaram gestantes com idade abaixo de 14 meses foram classificadas como super-precoces, e aquelas que ficaram gestantes com idade entre 14 e 16 meses foram classificadas como precoces.

Para os touros utilizados nesse estudo, inicialmente foi realizada análise de consistência dos dados, e descartado touros que tinha menos de três filhas no rebanho. Foi aplicado Teste de Qui-quadrado para verificar se a escolha do touro influenciou no resultado do diagnóstico de gestação e na classificação das novilhas nas categorias. Avaliou-se também o potencial genético dos touros pais das novilhas por meio de análise de variância e teste de comparação múltipla.

Realizou-se análise descritiva dos dados, empregando-se delineamento inteiramente casualizado, onde as variáveis foram comparadas pelo teste de Qui quadrado e Teste de Tukey, considerando 5\% de significância. Para processamento e análise dos dados foi utilizado o PROC GLM do programa SAS (Sas Institute, 2002).

\section{Resultados e Discussão}

A taxa de gestação de 184 novilhas da raça Nelore estudadas foi de $42 \%$. Gottschall \& Silva (2018), relataram que resultados de gestação em novilhas super precoces variaram entre $35 \%$ e $40 \%$, com média de $36,4 \%$ de gestação em novilhas de 14 meses de idade.

A ciclicidade reprodutiva de uma fêmea é uma consequência de uma série acumulativa de eventos hormonais e está mais associada ao peso corporal do que com a idade (Mousquer et al., 2014). Semmelmann et al. (2001) abordaram que o peso e a idade ao início da estação do acasalamento são importantes para o sucesso na gestação de novilhas, adiantando assim a sua concepção dentro do rebanho.

Na Tabela 1 estão descritas as médias, os desvios padrão e os valores mínimos e máximos para AOL, ACAB, peso ajustado no dia da IC, idade e índice de seleção para as novilhas estudadas.

Tabela 1. Valores médios das variáveis AOL, ACAB, peso ajustado, idade e índice de seleção das novilhas estudadas.

\begin{tabular}{lccc}
\hline \multicolumn{1}{c}{ Variável } & N & Média \pm Desvio Padrão & Mín. - Máx. \\
\hline $\mathrm{AOL}\left(\mathrm{cm}^{2}\right)$ & 183 & $53,45 \pm 5,54$ & $38,21-69,55$ \\
$\mathrm{ACAB}(\mathrm{mm})$ & 184 & $4,36 \pm 0,97$ & $2,25-7,71$ \\
Peso ajustado $(\mathrm{Kg})$ & 183 & $280,17 \pm 30,19$ & $184,25-342,17$ \\
Idade (meses) & 184 & $12,81 \pm 0,66$ & $10,45-13,94$ \\
Índice de seleção (meses) & 183 & $101,04 \pm 8,81$ & $76,31-126,19$ \\
\hline
\end{tabular}

Fonte: Dados da pesquisa.

Tabela 2 apresenta as características da AOL, ACAB, peso ajustado, idade e índice de seleção entre novilhas gestantes e não gestantes. 
Tabela 2. Valores médios das variáveis AOL, ACAB, peso ajustado, idade e índice de seleção das novilhas gestantes e não gestantes.

\begin{tabular}{|c|c|c|c|c|c|}
\hline \multirow{2}{*}{ Variável } & \multirow{2}{*}{$\mathbf{N}$} & Gestante & \multirow{2}{*}{$\mathbf{N}$} & \multirow{2}{*}{$\begin{array}{c}\text { Não gestante } \\
\overline{\mathbf{X}} \pm \mathrm{DP}\end{array}$} & \multirow{2}{*}{ Valor $\mathbf{p}$} \\
\hline & & $\overline{\mathbf{X}} \pm \mathbf{D P}$ & & & \\
\hline $\operatorname{AOL}\left(\mathrm{cm}^{2}\right)$ & 79 & $54,16 \pm 4,52$ & 105 & $52,92 \pm 6,16$ & 0,1332 \\
\hline $\mathrm{ACAB}(\mathrm{mm})$ & 79 & $4,40 \pm 0,82$ & 105 & $4,33 \pm 1,07$ & 0,6310 \\
\hline Peso ajustado (Kg) & 79 & $287,27 \pm 30,28^{\mathrm{A}}$ & 104 & $274,84 \pm 29,14^{\mathrm{B}}$ & $<0,0029$ \\
\hline Idade (meses) & 79 & $12,86 \pm 0,69$ & 105 & $12,76 \pm 0,65$ & 0,3266 \\
\hline Índice de seleção (meses) & 79 & $102,71 \pm 6,75^{\mathrm{A}}$ & 104 & $99,77 \pm 9,94^{\mathrm{B}}$ & $<0,024 *$ \\
\hline
\end{tabular}

A, B Valores seguidos por letras diferentes, na mesma linha, diferem $(\mathrm{p}<0,05)$. Fonte: Dados da pesquisa.

A AOL não apresentou diferença entre novilhas gestantes e não gestantes (p>0,05 - Tabela 2). Urbano et al. (2017) discorreram que os tecidos musculares de animais jovens se desenvolvem em ondas de crescimento específicas, e que animais mais velhos apresentam maior acúmulo de gordura na região da picanha, quando comparadas a animais mais jovens, o que indica diferença estrutural no desenvolvimento dos animais.

De forma semelhante o acabamento de carcaça foi independente do diagnóstico de gestação (p>0,05 - Tabela 2). Estudos indicam que a fêmea Nelore ovula após realizar certa quantidade de acúmulo de gordura em relação ao seu peso. Esse mecanismo favorece que o animal tenha reserva de energia, e também a ativação de algumas funções vitais como a reprodução (Castro et al., 2017). Ademais, Giraldo-Arana (2015) e Arana (2019), em experimento com novilhas da raça Nelore, não observaram diferenças na taxa de gestação para a espessura de gordura, semelhante ao encontrado neste estudo. Esses autores acrescentaram que essa variável pode estar atrelada a algum fator genético, como formação do grupo contemporâneo, que vem sendo melhorado com foco em fertilidade há 30 anos, onde sempre procurou fixar genes de fertilidade, concordando com os animias utilizados nesse estudo, uma vez que pertecem a um mesmo grupo de manejo, para que as avaliações do programa de melhoramento utilizado sejam as mais fidedignas possíveis.

Arana (2019), relatou que a variável ACAB pode haver interferência de fator nutricional, onde os animais fortemente suplementados teriam melhor desempenho. Neste estudo, as novilhas foram mantidas a pasto, com suplementação de manutenção, excluindo-se assim possível interferência do fator nutricional.

D’Occhio et al. (2018) reportaram que o ganho de peso pré desmana é de suma importância para as fases subsequentes do desenvolvimento do animal como futura reprodutora bovina, na fase pós-desmama, para novilhas manterem ótima condição corporal e acumular gordura, precisam ganhar entre 600 e 700 g/dia. As novilhas estudadas apresentaram, em média 600 gramas de ganho de peso diário, encontrando-se em acúmulo de gordura, segundo D’Occhio et al. (2018). Urbano et al. (2017) reafirmaram que animais que realizam deposição de gordura subcutânea são mais precoces. O crescimento segue uma ordem fisiológica onde crescem primeiro os tecidos nervosos na sequência ósseo e muscular, e por último tecido adiposo (Owens et al., 1993; Paulino et al., 2009).

Observou-se que novilhas gestantes foram mais pesadas que novilhas não gestantes ( $\mathrm{p}<0,05-$ Tabela 2$)$. O peso da fêmea é fator crucial para que a mesma venha gestar um concepto, e chegue ao final da gestação com sucesso (Semmelmann et al., 2001). Funston et al. (2012) relataram que o peso está diretamente correlacionado ao início da puberdade de novilhas Nelore, e ainda que femêas desmamadas com $51 \%$ do peso adulto chegam primeiro ao início da ciclicidade. Adicionamente, Mercadante et al. (2003), estudando novilhas Nelore selecionadas para alto peso corpóreo, constataram que esses animais não apresentaram prejuízo no desempenho reprodutivo. Já Kirkpatrick et al. (2018) encontraram correlação positiva entre todas as características correlacionadas a fertilidade, precocidade e carcaça, exceto para acabamento, que não se correlacionou com 
nenhuma outra característica. Semmelmann et al. (2001) obtiveram 20\% de gestação em novilhas Nelore aos 18 meses de idade pesando $262 \mathrm{Kg}$.

De acordo com Silva et al. (2009), novilhas Nelore recriadas extensivamente, atingiram puberdade aos 22 meses de idade, com $312 \mathrm{Kg}$. Neste estudo, a média de peso foi de 280,17 Kg ajustado ao dia da IC (30 dias antes do início da sincronização), estimando-se que no dia da sincronização as mesmas se encontravam com peso médio ajustado de $300 \mathrm{~kg}$. $\mathrm{O}$ peso ajustado no dia IC influenciou a taxa de gestação, onde novilhas mais pesadas tiveram maior porcentagem de gestação ( $<<0,05$ - Tabela 2). Observou-se ainda, que o índice de seleção para precocidade sexual influenciou a taxa de gestação ( $<<0,05$ - Tabela 2), possivelmente por se tratar de um índice cuja composição possui forte ponderação para a característica peso, que apresentou, isoladamente efeito significativo.

A Tabela 3 apresenta a porcentagem de novilhas gestantes e não gestantes entre as categorias estudadas, que apresentaram taxa de gestação semelhante $(\mathrm{p}>0,05)$. Este resultado poderia ser explicado pelo baixo número de novilhas na Categoria C (15,76\% - 29 novilhas) em detrimento ao número maior de novilhas nas categorias A e B (84,24\% - 155 novilhas), indicando um rebanho em processo de seleção para precocidade sexual já em andamento. O início da vida reprodutiva do animal se dá no momento em que aparece o primeiro estro fértil, que pode ser influenciado por fatores genéticos, ambientais, nutricionais, raciais e estação de nascimento. Animais zebuínos tendem a ser mais tardios quando comparados a animais taurinos (Rodrigues et al., 2002).

Tabela 3. Número de animais e porcentagem de novilhas gestantes e não gestantes entre as categorias estudadas.

\begin{tabular}{cccc}
\hline Categoria & Gestante & Não gestante & Total \\
\cline { 2 - 4 } & N (\% gestação) & N (\% gestação) & N (\% gestação) \\
\hline Categoria A & $20(48,78)$ & $21(52,22)$ & $41(22,28)$ \\
Categoria B & $49(42,98)$ & $65(57,02)$ & $114(61,96)$ \\
Categoria C & $10(34,48)$ & $19(65,52)$ & $29(15,76)$ \\
\hline Total & $79(42,93)$ & $105(57,07)$ & $184(100)$ \\
\hline
\end{tabular}

Fonte: Dados da pesquisa.

Não se observou diferença significativa da AOL e ACAB entre as categorias estudadas ( $p>0,05$ - Tabela 4). No entanto, o peso ajustado, idade e índice de seleção apresentaram diferenças entre as novilhas, nas diferentes categorias ( $\mathrm{p}<0,05$ - Tabela 4). Observou-se que a maturidade dos órgãos reprodutivos está diretamente associada à idade e ao peso das novilhas. Considerando que as avaliações de AOL e ACAB foram realizadas quando as novilhas já se encontravam em média com 20 meses de idade, pode ter havido redução da relação da idade com a maturidade do aparelho reprodutor. Ademais, o peso influencia significativamente na puberdade da fêmea bovina, devendo-se desmamar os animais com $50 \%$ do seu peso adulto, através de seleção genética, a fim de reduzir custos e melhorar a eficiência reprodutiva em fêmeas super precoces (Funston et al., 2012). 
Tabela 4. Valores médios das variáveis AOL, ACAB, peso ajustado, idade e índice de seleção entre as categorias estudadas.

\begin{tabular}{|c|c|c|c|c|c|c|c|}
\hline \multirow{2}{*}{ VARIÁVEL } & \multirow{2}{*}{$\mathbf{N}$} & Categoria A & \multirow{2}{*}{$\mathbf{N}$} & Categoria B & \multirow{2}{*}{$\mathbf{N}$} & Categoria C & \multirow{2}{*}{ Valor $\mathbf{p}$} \\
\hline & & $\bar{X} \pm \mathbf{D P}$ & & $\bar{X} \pm \mathbf{D P}$ & & $\bar{X} \pm \mathrm{DP}$ & \\
\hline $\mathrm{AOL}\left(\mathrm{cm}^{2}\right)$ & 41 & $53,98 \pm 5,86$ & 114 & $53,61 \pm 5,28$ & 29 & $52,12 \pm 6,04$ & 0,3446 \\
\hline $\mathrm{ACAB}(\mathrm{mm})$ & 41 & $4,56 \pm 1,15$ & 114 & $4,32 \pm 0,84$ & 29 & $4,24 \pm 1,15$ & 0,3064 \\
\hline Peso ajustado (Kg) & 41 & $292,23 \pm 22,47^{\mathrm{A}}$ & 113 & $284,65 \pm 26,77^{\mathrm{A}}$ & 29 & $245,52 \pm 28,34^{\mathrm{B}}$ & $<0,001$ \\
\hline Idade (meses) & 41 & $12,90 \pm 0,70^{\mathrm{A}}$ & 114 & $12,86 \pm 0,65^{\mathrm{A}}$ & 29 & $12,45 \pm 0,56^{\mathrm{B}}$ & $<0,0070$ \\
\hline $\begin{array}{l}\text { Índ. seleção } \\
\text { (meses) }\end{array}$ & 41 & $103,89 \pm 9,79^{\mathrm{A}}$ & 113 & $101,10 \pm 7,85^{\mathrm{A}}$ & 29 & $96,77 \pm 9,51^{\mathrm{B}}$ & $<0,0035$ \\
\hline
\end{tabular}

A, B Valores seguidos por letras diferentes, na mesma linha, diferem $(\mathrm{p}<0,05)$. Fonte: Dados da pesquisa.

Novilhas das categorias A e B apresentaram peso ajustado semelhante $(p>0,05)$ e superior aquelas da categoria C $(\mathrm{p}<0,05)$, como mostra a Tabela 4. Esses valores são considerados elevados para a raça Nelore nesta idade, o que contribuiu para alcance da maturidade sexual das novilhas destas categorias, uma vez que a fêmea bovina não consegue entrar em puberdade até que acumule um mínimo de 2,9 mm de gordura (ideal 5,8 mm). A deposição de gordura está correlacionada à massa corporal, que denota o acúmulo energético necessário para suprir exigências de gestação e lactação. Assim sendo, o escore corporal desencadeia um sinal endógeno associado ao início da puberdade (Castro et al., 2017). No entanto, no presente estudo, não se observou diferença para o ACAB, talvez por representar um fator genético.

Staples (1998) relatou que novilhas com maior acúmulo de gordura na picanha, possuem os órgãos reprodutivos mais desenvolvidos, o que pode acarretar melhor desempenho reprodutivo. Castro et al. (2017) relataram que novilhas com maior deposição de gordura apresentaram maior índice de gestação, indicando assim, que este fator indica maior precocidade. Estes autores ainda salientaram que para se reduzir a faixa etária das fêmeas expostas à estação de monta, deve-se aumentar o acúmulo de gordura na picanha. Mamede (2015) relatou que AOL sinaliza influência negativa na idade ao primeiro parto (IPP), porém apresenta maior peso na carcaça. Laeflet (2001) concluiu que o ACAB está ligado a condição corporal do animal, sendo um ponto chave na redução da idade ao primeiro acasalamento, onde os animais com maior deposição de gordura tendem a ser mais precoces, apresentando trato reprodutivo mais desenvolvido com menor faixa etária.

De forma semelhante, a idade também influenciou a categoria das novilhas estudadas $(\mathrm{p}<0,05)$, onde novilhas mais jovens foram as novilhas acíclicas (Tabela 4). No entanto, é possível inferir que novilhas mais jovens de maior peso dentro destas categorias deveriam ser as preferidas dentro do processo seletivo, por possuir genes que conferem simultaneamente capacidade para ganho de peso e maturidade sexual. Conforme Krappa (2018), à medida que os animais mais jovens vão sendo exposto ao manejo reprodutivo, o desempenho não depende somente do ambiente. Neste estudo, observou-se que as novilhas experimentais foram dependentes das condições do ambiente, uma vez que quanto mais favoráveis as condições para o maior ganho de peso pré e pós desmame, maior será a velocidade de maturidade sexual.

A idade não teve correlação com o peso ajustado, AOL e ACAB ( $p>0,05$ - Tabela 5), podendo sugerir a presença de novilhas jovens e mais pesadas, e vice-versa. Ressalta-se que na Tabela 4, as variáveis peso ajustado e idade, foram determinantes para a maturidade sexual das novilhas inseridas nas categorias A e B. No entanto, como não se verificou correlação entre essas variáveis (peso ajustado x idade), é possível inferir que há novilhas de maior precocidade sexual nestas categorias, podendo ser identificadas e selecionadas. 
Tabela 5. Correlações de Pearson entre as variáveis de AOL, ACAB, peso ajustado, idade e índice seleção das novilhas estudadas.

\begin{tabular}{cccccc}
\hline & Peso ajustado & AOL & ACAB & Índice Seleção & Idade \\
\hline Peso ajustado & 1 & - & - & - & - \\
AOL & $0,37^{*}$ & 1 & - & - & - \\
ACAB & 0,11 & $0,24^{*}$ & 1 & - & - \\
Índice Seleção & $0,67^{*}$ & $0,63^{*}$ & $0,76^{*}$ & 1 & - \\
Idade & $-0,04$ & $-0,08$ & 0,07 & 0,00 & 1 \\
\hline
\end{tabular}

* Valores seguidos por asterístico apresentaram diferença estatística $(\mathrm{p}<0,05)$. Fonte: Dados da pesquisa.

O índice de seleção adotado apresentou correlação com peso ajustado, AOL e ACAB (p<0,05 - Tabela 5), pelo fato de possuir estas variáveis na sua formação. Observa-se também correlação entre peso ajustado e AOL (p<0,05 - Tabela 5), indicando que animais mais pesados tendem a ter maior valor de AOL. Contudo, como o valor de correlação é relativamente baixo (Tabela 5), torna-se necessário classificar e identificar as fêmeas que de fato possuem maior peso, maior AOL e menor idade. Este processo seletivo conduziria a fêmeas com maior capacidade de ganho de peso, maior AOL e precocidade sexual.

Nos critérios de seleção, com maior representatividade nos programas de melhoramento, cita-se que o peso a desmama apresenta correlação direta com o peso final do animal (Ferraz Filho et al., 2002; Malhado et al., 2002). Porém esta característica é influenciada diretamente por diferentes fatores, como habilidade materna (Meyer, 1992). Quanto mais rápido o desenvolvimento dos animais, menor será o ciclo de produção e consequentemente menor custo de produção (Marcondes et al., 2000). Assim sendo, o ganho médio de peso auxilia na escolha de animais mais precoces (Marcondes et al., 2000; Garnero et al., 2001; Pereira et al., 2001). Porém a gestação é uma característica binária onde o sucesso é novilhas gestantes após expostas a estação de monta, e fracasso é a taxa de insucesso, a qual as mesmas não se tornam gestantes. Outro fato importante é que tende a ter herdabilidade de moderada a alta (Silva et al., 2003; Silva et al., 2005).

A escolha do touro não influenciou a taxa de gestação ( $p>0,05)$, demonstrando que há fatores mais relevantes que influenciaram na taxa de gestação das novilhas. Dessa forma, os touros utilizados no estudo, apresentaram méritos genéticos semelhantes para as características reprodutivas em estudo.

Ferraz et al. (2018) relataram que a escolha do sêmen é a mais efetiva e barata ferramenta para redução de seleção e a puberdade em novilhas com herdabilidade na progênie, e funciona como ferramenta onde as Dep's são de alta influencia na idade a puberdade, sendo as escolhas de touros precoces uma ferramenta efetiva no processo de seleção. Sendo assim, a escolha do pai e da mãe na definição do acasalamento, são de que pais precoces dão origem a descendentes também precoces (Guimarães et al., 2011).

Não se observou diferenças para a AOL, ACAB e índice de seleção entre novilhas precoce e super precoces (p>0,05 Tabela 6). No entanto, novilhas super precoces ficaram gestantes com peso ajustado e idade inferiores que as novilhas precoces $(\mathrm{p}<0,05$ - Tabela 6). Arana et al. (2019) relataram que o peso e o biotipo da novilha Nelore exercem influência na puberdade e na primeira estação de monta. Animais com maior ganho diário de peso e melhor distribuição na carcaça, tanto na desmama como no sobreano, apresentam melhor desempenho na primeira estação de monta. 
Tabela 6. Valores médios das variáveis AOL, ACAB, peso ajustado, idade e índice de seleção em novilhas precoce e superprecoce.

\begin{tabular}{|c|c|c|c|c|c|}
\hline \multirow{2}{*}{ Variável } & \multicolumn{2}{|c|}{ Precoce } & \multicolumn{2}{|c|}{ Superprecoce } & \multirow{2}{*}{ Valor $\mathbf{p}$} \\
\hline & $\bar{X} \pm \mathrm{DP}$ & Min. - Max. & $\bar{X} \pm \mathbf{D P}$ & Min. - Max. & \\
\hline $\operatorname{AOL}\left(\mathrm{cm}^{2}\right)$ & $53,61 \pm 4,50$ & $42,20-63,92$ & $55,02 \pm 4,50$ & $44,70-62,92$ & 0,1782 \\
\hline $\mathrm{ACAB}(\mathrm{mm})$ & $4,44 \pm 0,81$ & $2,25-5,93$ & $4,34 \pm 0,85$ & $2,64-6,01$ & 0,6012 \\
\hline Peso ajustado $(\mathrm{Kg})$ & $298,51 \pm 28,13^{\mathrm{A}}$ & $223,58-346,00$ & $277,44 \pm 30,26^{\mathrm{B}}$ & $196,01-321,74$ & $<0,0023$ \\
\hline Idade (meses) & $14,45 \pm 0,25^{\mathrm{A}}$ & $14,04-15,02$ & $13,24 \pm 0,47^{\mathrm{B}}$ & $11,57-13,94$ & $<0,0001$ \\
\hline $\begin{array}{l}\text { Índ. seleção } \\
\text { (meses) }\end{array}$ & $102,54 \pm 6,81$ & $85,01-114,76$ & $102,98 \pm 6,76$ & $88,16-13,94$ & 0,7775 \\
\hline
\end{tabular}

A, B Valores seguidos por letras diferentes, na mesma linha, diferem $(\mathrm{p}<0,05)$. Fonte: Dados da pesquisa.

A condição reprodutiva não foi determinante para classificação das novilhas como precoce ou super-precoce ( $p>0,05$ - Tabela 7). A indução do estro através de protocolos de indução pode trazer a ciclicidade animais que não se encontravam em estro (Araújo et al., 2007).

Tabela 7. Números e porcentagem de novilhas precoce ou superprecoce de acordo com a categoria estudada.

\begin{tabular}{cccc}
\hline \multirow{2}{*}{ Categoria } & Precoce & Superprecoce & Total \\
\cline { 2 - 4 } & $\mathbf{N}(\%)$ & $\mathbf{N}(\%)$ & $\mathbf{N}(\%)$ \\
\hline Categoria A & $14(70,00)$ & $6(30,00)$ & $20(25,32)$ \\
Categoria B & $31(63,27)$ & $18(36,73)$ & $49(62,03)$ \\
Categoria C & $3(30,00)$ & $7(70,00)$ & $10(12,66)$ \\
\hline Total & $48(60,76)$ & $31(39,24)$ & $79(100)$ \\
\hline
\end{tabular}

Fonte: Dados da pesquisa.

\section{Conclusão}

Nas condições desse estudo, conclui-se que as novilhas Nelore apresentaram elevada precocidade sexual, eficiente para obtenção para novilhas precoces e super-precoce, respectivamente, sendo aquelas que apresentaram maior peso, no início do desafio, tenderam a ser mais precoces, indicando a importância desta característica em sistemas de produção.

No entanto, estudos complementares sobre processo de seleção para precocidade sexual, visando obtenção de novilhas cada vez mais precoces, com maior peso e menor idade à primeira concepção e parto, serão continuadamente desenvolvidos visando incrementar os sistemas de produção de gado de corte, sobretudo aqueles inseridos no Estado do Acre.

\section{Referências}

Abud, L. J. Fatores que influenciam o início da puberdade. (2011). Disciplina: seminários aplicados (Pós - Graduação em Ciência Animal), Universidade Federal de Goiás, Goiânia.

Alencar, M. M. Critérios de seleção em bovinos de corte. (2002). In: Curso de Melhoramento de Gado de Corte- Geneplus, Embrapa Pecuária Sudeste, Campo Grande, MS.

Arana, D. G. Comparação de critérios para predição da precocidade em novilhas nelore. (2019). Tese (Doutor em Ciência Animal), Universidade Estadual Paulista (Unesp), Araçatuba, São Paulo.

Araújo, A. C. C., Nonato, M. S. \& Bezerra, A. R. A. (2007). Indução da puberdade em novilhas com uso de hormonioterapia. Ciência Animal, 17(2), 83-89. 10.34117/bjdv5n11-115. 
Artmann, T. A., Toma, H. S., Pinheiro, J. N., Romero, J., Carvalho, A. M. \& Monteiro Toma, C. D. (2014). Eficiência produtiva brasileira e sua associação ao melhoramento genético animal. Revista Científica de Medicina Veterinária, (22). http://faef.revista.inf.br/.

Associação Brasileira das Indústrias Exportadoras de Carnes. (2018). Perfil da Pecuária no Brasil. Relatório Anual, 1-46, São Paulo.

Barbosa, F.A., Filho, B. S. S., Merry, F. D., Azevedo, H. O., Costa, W. L. S., Coe, M. T., Batista, E. L. S., Maciel, T. G., Sheepers, L. C., Oliveira, A. R. \& Rodrigues, H. O. (2015). Cenários para a pecuária de corte na Amazônia. Belo Horizonte: UFMG. 146.

Boligon, A. A. \& Albuquerque. (2010). Correlações genéticas entre escores visuais e características reprodutivas em bovinos Nelore usando inferência bayesiana. Pesquisa Agropecuária Brasileira, 45, (12), 1412-1418. 10.1590/S0100-204X2010001200011.

Brasil. Ministério da Agricultura, Pecuária e Abastecimento. (2018). Dados de rebanho bovino e bubalino no Brasil. https://www.gov.br/.

Cartwright, T.C. (1970). Selection criteria for beef cattle for the future. Journalof Animal Science, 30, 706- 711. 10.2527/jas1970.305706x.

Carvalho, T. B. \& Zen, S. D. (2017). A cadeia de Pecuária de Corte no Brasil: evolução e tendências. Revista iPecege, 3, (1), 85-99. 10.22167/r.ipecege.2017.1.85.

Castro, L. M., Claudio, U. M., Fernando, O. C., Fernando, S. B. R., Ludmilla, C. B., Luis, C. R. Q., Marcos, F. O. C. \& Raysildo, B. L. (2017). Analise Discriminante entre ocorrência de gestação precoce e características de carcaça em bovinos da raça nelore. In:Congresso Zootec Santos, São Paulo.

D'oochio, M. J., Baruselli, P. S. \& Campanille, G. (2018). Influence of nutrition, body condition, and metabolic status on reproduction in female beef cattle: A review. Theriogenology, 125, 277-284. 10.1016/j. theriogenology.2018.11.010.

Fernandes, P. B., Marques, K. O., Araujo Neto, F. R., De Oliveira, D. P., Hurtado-Lugo, N. A., Aspilcueta-Borquis, R.R. \& Tonhati, H. (2016). Geneticquantitative study of the first-Service pregnancy probability of murrah heifers. Reproduction Domestic Animals., 51,428-434. 10.1111/rda.12697.

Ferraz Filho, P. B., Ramos, A. A., Silva, L. O. C., Souza, J. C. \& Alencar, M. M. (2002). Herdabilidades e correlações genéticas, fenotípicas e ambientais para pesos em diferentes idades de bovinos da raça Tabapuã. Archives of Veterinary Science., 7, (1), 75-79. 10.5380/avs. v7i1.3970.

Ferraz, M. V. C., Pires, A. V., Santos, M. H., Silva, R. G., Oliveira, G. B., Polizel, D. M., Biehl, M. V., Sartori, R. \& Nogueira, G. P. (2018). A combination of nutrition qnd genética is able to reduce age at puberty in Nelore heifers to below 18 months. Animal., 12, (3), 569-574. 10.1017/S1751731117002464.

Funston, R. N., Martin, J. L., Larson, D. M. \& Roberts, A. J. (2012). Physiology and endocrinology symposium: Nutritional aspects of developing replacement heifers. Journal of Animal Science., 90, (4), 1166-1171. 10.2527/jas.2011-4569.

Garnero, A. D. V., Lôbo, R. B., Bezerra, L. A. F., Framartino, L. A. \& Oliveira, H. N. (2001). Comparação entre Alguns Critérios de Seleção para Crescimento na Raça Nelore. Revista Brasileira de Reprodução Animal., 30, (3), 714-718. 10.1590/S1516-35982001000300016.

Giraldo-Arana, D. (2015). Efeitos da somatotrofina recombinante bovina na puberdade de novilhas Nelore. Dissertação (Mestrado - Faculdade de Medicina Veterinária). Universidade Estadual Paulista julho de Mesquita Filho.

Gottschall, C. S. \& Silva, L. R. (2018). Taxa de prenhez de novilhas de corte submetidas à IATF com protocolo a base de benzoato de estradiol ou Ovsynch modificado. PUBVET., 12, (9), 1-6. https://www.pubvet.com.br/.

Guimarães, J. D., Guimarães, S. E. F., Siqueira, J. B., Pinho, R. O., Eler, J. P., Ferraz, J. B. S., Silva, M. R. \& Borges, J. C. (2011). Seleção e manejo reprodutivo de touros zebu. Revista Brasileira de Zootecnia.,40, 379-388. http://sbz.org.br/new/pt/.

Jorge Junior, J., Pita, F. V. C., Fries, L.A. \& Albuquerque, L. G. (2001). Influência de Alguns Fatores de Ambiente sobre os Escores de Conformação, Precocidade e Musculatura à Desmama em um Rebanho da Raça Nelore. Revista Brasileira de Zootecnia., 30, (6). 10.1590/S1516-35982001000700006.

Kirkpatrick, T., Reed, J., May, N., Walter, L., Mcevers, T., Hutcheson, J. \& Lawrence, T. (2018). PSV-12 Allometric growth of serially harvested calf-fed Holstein steers supplemented zilpaterol hydrochloride. Journal of Animal Science., 96, 45-46. 10.1093/jas/sky404.102.

Krapp, A. (2018). Parâmetros genéticos para características de carcaça, peso corporal e idade ao primeiro parto em bovinos da raça Nelore. (Programa de PósGraduação em Zootecnia), Universidade Federal de Mato Grosso, Instituto de Ciências Agrárias e Ambientais Sinop, Mato Grosso.

Lanna, D. P. \& Packer, I. U. (1997). A produtividade da vaca Nelore. In: Simpósio: O Nelore do Século XXI., 4, 73-86.

Leaflet, A. S. (2001). Relationship between body composition and reproduction in heifers. Iwoa State University: Ames.

Malhado, C. H. M., Martins Filho, R. Lôbo, R. N. B., Facó, O. \& Souza, J. C. (2002). Efeitos ambientais que influenciam a velocidade de crescimento em bovinos Nelore na região Nordeste. In: Reunião Anual da Sociedade Brasileira de Zootecnia, 39.

Mamede, M. M. S. (2015). Análise genética para maciez de carne e suas relações com as características produtivas em bovinos nelore mocho. Tese (Doutorado em Ciência Animal), Universidade Federal de Goiás, Goiânia.

Marcondes, C. R., Bergmann, J. A. G., Eler, J. P., Ferraz, J. B. S., Pereira, J. C. C. \& Penna, V. M. (2000). Análise de alguns critérios de seleção para características de crescimento na raça Nelore. Arquivo Brasileiro de Medicina Veterinária e Zootecnia., 52, (1),83-89. 10.1590/S0102-09352000000100018.

Mercadante, M. E. Z., Packer, I. U., Razook, A. G., Cyrillo, J. N. S. G. \& Figueiredo, L. A. (2003). Direct and correlated responses to selection for yearling weight on reproductive performance of Nelore cows Journal of Animal Science., 81, 376-384. 10.2527/2003.812376x.

Meyer, K. (1992). Variance components due to direct and maternal effects for growth traits of Australian beef cattle. Livestock Production Science., 31, (3-4), 179-204. 10.1016/0301-6226(92)90017-X. 
Mousquer, C. J., Fernandes, F. F. D., Fernandes, G. A. \& Castro, W. J. R. (2014). Desempenho reprodutivo de matrizes Nelore. PUBVET., 8, (3),15. https://www.pubvet.com.br.

Oaigen, R. P., Barcellos, J. O. J., Canozzi, M. E. A., Soares, J. C. R., Canellas, L. C., Alves, C. O., Tavares, H. R. \& Costa, F. M. (2013). Competitividade inter-regional de sistemas de produção de bovinocultura de corte. Ciência Rural., 43, (8), 1489-1495. 0.1590/S0103-84782013000800024.

Owens, F.N., Dubeski, P. \& Hanson, C.F. (1993). Factors that alter the growth and development of ruminants. Journal of Animal Science., 71, (11), 3138 3150. 10.2527 / 1993.71113138x

Paulino, P. V. R., Valadares Filho, S. C., Detmann, E., Valadares, R. F. D., Fonseca, M. A. \& Marcondes, M.I. (2009). Deposição de tecidos e componentes químicos corporais em bovinos Nelore de diferentes classes sexuais. Revista Brasileira de Zootecnia., 38, (12), 2516-2524. 10.1590/S151635982009001200030 .

Pereira, E., Eler, J. P. \& Ferraz, J. B. S. (2001). Análise genética de algumas características reprodutivas e suas relações com o desempenho ponderal na raça Nelore. Arquivo Brasileiro de Medicina Veterinária e Zootecnia., 53, (6), 720-727. 10.1590/S0102-09352001000600019.

Pereira, A., S., Shitsuka, D., M., Parreira, F., J. \& Shitsuka, R. (2018). Metodologia da pesquisa científica. [free e-book]. Santa Maria. Ed. UAB/NTE/UFSM. https://repositorio.ufsm.br/.

Rodrigues, H. D., Kinder, J. E. \& Fitzpatrick, L. A. (2002). Estradiol regulation of luteinizing hormone secretion in heifers of two breed types that reach puberty at different ages. Biology of Reproduction., 66, $603-609.10 .1095 /$ biolreprod66.3.603.

Rosa, A. N. F., Oliveira, M. G. R. \& Suleiman, K. (2016). O valor do zebu. AgroAnalysis., 36, (7), 23-24. http://bibliotecadigital.fgv.br/.

Sá, C. P., Andrade, C. M. S. \& Valentim, J. F. (2010). Análise econômica para a pecuária de corte em pastagens melhoradas no Acre. Embrapa Acre, Rio Branco.

Semmelmann, C. E. N., Lobato, J. F. \& Rocha, M. G. (2001). Efeito de sistemas de alimentação no ganho de peso e desempenho reprodutivo de novilhas Nelore acasaladas aos 17/18 meses. Revista Brasileira Zootécnica., 30, (3), 835-843. 10.1590/S1516-35982001000300032.

Silva, F. M. B., Lopes, D. T., Ferraz, H. T., De Oliveira Viu, M. A., De Souza Ramos, D. G., Saturnino, K. C., Fontana, C. A. P., Silva, J. M. A. \& Leso, F. V. (2018). Estratégias para antecipação da puberdade em novilhas Bos taurus indicus pré-púberes. PUBVET., 12, (12), 136. http://www.pubvet.com.br/.

Silva, J. A., Albuquerque, L. G., Sesana, R. C., et al. (2005). Correlação genética entre prenhez de novilhas e perímetro escrotal em animais Nelore utilizando inferência Bayesiana. In: Reunión de La Asociación Latinoamericana de Producción Animal. Anais... Tampico: Asociación Latinoamericana de Producción Animal.

Silva, J. A. II V., Van Melis, M. H., Eler, J. P. \& Ferraz, J. B. S. (2003). Estimação de parâmetros genéticos para probabilidade de prenhez aos 14 meses e altura na garupa em bovinos da raça Nelore. Revista Brasileira de Zootecnia., 32, (5), 1141-1146. 10.1590/S1516-35982003000500014.

Silva, L. F. P. E., Carvalho, M. V. \& Diniz- Magalhães, J. (2009). Crescimento e puberdade de novilhas zebuínas. In: Santos, M. V., Rennó, F. P., Silva, L. F. P. \& R., A. Novos desafios da pesquisa em nutrição e produção animal. Pirassununga.

Siqueira, R. L. P. G., Oliveira. J. A., Lôbo, R. B., Bezerra, L. A. F. \& Tonhati, H. Análise da variabilidade genética aditiva e de características de crescimento da raça Nelore. Revista Brasileira de Zootecnia., 32, (1), 99-105. 10.1590/S1516-35982003000100013.

Souza, E. M. D., Milagres, J. C., Silva, M. D. A., Regazzi, A. J. \& Castro, A. G. C. (1995). Influências genéticas e de meio ambiente sobre a idade ao primeiro parto em rebanhos de Gir leiteiro. Revista Sociedade Brasileira de Zootecnia., 24, (6), 926-935.

Staples, C. R., Burke, J. \& Thatcher, W. W. (1998). Shyposium: optimizing energy nutrition for reproduction dairy cows. Journal Dairy Science., 81, (3), 865871.

Statistical Analysis System - Sas. (2002). User's guide. Cary: SAS Institute.

Urbano, S. A., Ferreira, M. A., Rangel, A. H. N., Lima Júnior, D. M., Andrade, R. P. X. \& Novaes, L. P. (2017). Lamb feeding strategies during the preweaning period in intensive meat production systems. Tropical and Subtropical Agroecosystems., $20, \quad(1), \quad 49 \quad-\quad 63$. https://www.redalyc.org/articulo.oa?id=93950595001. 